\title{
El discurso de la calidad educativa: un análisis crítico
}

\section{Leonardo Colella}

Conicet (Argentina)

leonardojcolella@yahoo.com.ar

\section{Rocío Díaz-Salazar}

Universidad de Buenos Aires (Argentina) rocbelds@hotmail.com

\section{Resumen}

El artículo indaga desde la perspectiva del análisis del discurso, el sintagma "calidad educativa" en diversos documentos de politica educativa de finales del siglo XX, con el propósito de dilucidar el sentido politico-social de la educación. Sostiene que el concepto de "calidad educativa" es un punto privilegiado de reflexión, en tanto opera como una sintesis del conjunto de ideas mediante el que se desea establecer las propiedades de la educación. Este trabajo establece un análisis de la configuración del discurso de la calidad de la educación como forma precaria y relativa de fijación del sentido, que se halla vinculado directamente al orden social.

\section{Palabras clave}

Calidad de la educación, reforma de la educación, educación y desarrollo, evaluación de la educación, teoría de la educación (Fuente: Tesauro de la Unesco).

Recepción: 2014-12-10 | Envío a pares: 2015-02-21 | Aceptación por pares: 2015-07-28 | Aprobación: 2015-08-10 DOI: 10.5294/edu.2015.18.2.6

Para citar este artículo / To reference this article / Para citar este artigo

Colella L., Díaz-Salazar R. (2015). El discurso de la calidad educativa: un análisis crítico. Educ. Educ., 18 (2), 287-303. DOI: 10.5294/ edu.2015.18.2.6 


\title{
The Discourse on Quality of Education: A Critical Analysis
}

\begin{abstract}
This article looks at the phrase "quality education" in a number of documents on education policy at the end of the twentieth century. It does so from the standpoint of analyzing the discourse on this point and to clarify the political and social meaning of education. The author argues the concept of "quality education" is a privileged point of reflection and one that combines all the ideas we want the properties of education to reflect. This study considers the pattern of the discourse on the quality of education as a precarious and relative way to assign meaning that is linked directly to the social order.
\end{abstract}

\section{Keywords}

Quality of education, education reform, education and development, evaluating education, educational theory (Source: Unesco Thesaurus). 


\section{O discurso da qualidade educativa: uma análise crítica}

Resumo

Este artigo indaga, sob a perspectiva da análise do discurso, acerca do sintagma "qualidade educativa" em diversos documentos de política educativa do final do século XX, com o objetivo de elucidar o sentido político-social da educação. Sustentase que o conceito de "qualidade educativa" é um ponto privilegiado de reflexão enquanto opera como uma sintese do conjunto de ideias mediante o qual se pretende estabelecer as propriedades da educação. Este trabalho estabelece uma análise da configuração do discurso da qualidade da educação como forma precária e relativa de fixação do sentido, que se encontra vinculado diretamente à ordem social.

\section{Palavras chave}

Qualidade da educação, reforma da educação, educação e desenvolvimento, avaliação da educação, teoria da educação. (Fonte: Tesauro da Unesco). 


\section{Introducción}

El objetivo de este artículo es explorar la "esencia" y la "eficacia" propias del discurso de la calidad de la educación, indagando por los desplazamientos de sentido que tuvo el concepto de "calidad educativa" en diversos documentos de política educativa. Sostenemos que la indagación sobre los sentidos atribuidos al concepto nos permitirá ir descubriendo las propiedades que se le atribuyen a la educación y ello, a su vez, nos permitirá dilucidar los sentidos sociales y políticos que sustentan determinada concepción de la misma. En este sentido, nuestra labor no pretende ser meramente una descripción de elementos enunciados en los documentos de políticas educativas, sino un análisis de la configuración del discurso de la calidad de la educación como forma precaria y relativa de fijación del sentido que acompaña cierto orden social. Para realizar este trabajo profundizaremos en la teoría del discurso de Ernesto Laclau y Chantal Mouffe.

De este modo, la investigación considerará el análisis del discurso como una herramienta teórico-metodológica sustentada, principalmente, en la búsqueda de regularidades discursivas. En tal sentido, debemos señalar que si bien nuestra indagación supone la elaboración de un corpus de documentos de política educativa de organismos e instituciones regionales e internacionales, este se conformará en forma simultánea con su análisis.

No obstante, es preciso señalar que para la selección de los documentos tendremos en cuenta aquellos organismos regionales e internacionales cuyas enunciaciones han tenido una fuerte incidencia en América Latina. Asimismo, la elección del material empírico se sustenta en el carácter técnico y normativo que asume el sintagma "calidad educativa" en estos documentos.

El concepto "calidad educativa" se ubica en el centro del discurso de la calidad de la educación. Siguiendo la teoría que plantean Laclau y Moufee (2011), podemos definir al "discurso" como una to- talidad estructurada resultado de una práctica articulatoria. Esta práctica radica en establecer una relación entre diversos elementos cuya consecuencia es la modificación de la identidad de estos. La articulación de los elementos al interior del discurso los convierte en momentos. Con este término, los autores hacen alusión a su inclusión dentro de un sistema de posiciones diferenciales en el que la identidad de los elementos es siempre relacional. Por su parte, el sistema no debe ser pensado como la expresión de un principio subyacente exterior a sí mismo. Consideramos al discurso como una totalidad articulada de carácter contingente. Esto significa que "la articulación es una práctica discursiva que no tiene un plano de constitución a priori o al margen de la dispersión de los elementos articulados" (p. 149), y que "la fijación de los elementos en momentos no es nunca completa" (p. 144). Por esta razón, los autores sostienen que "una totalidad discursiva nunca existe bajo la forma de una positividad simplemente dada y delimitada" (p. 150). Ello implica que hay un exterior discursivo constituido por otros discursos que vulneran a nuestro discurso de la calidad educativa, deformándolo e impidiendo su sutura. Ese exterior recibe el nombre de campo de la discursividad y constituye un exceso de sentido que subvierte cualquier intento de fijación de sentido último por parte de un discurso. De este modo, toda configuración de posiciones diferenciales no debe ser pensada como un sistema de momentos necesarios de una ley inmanente, sino como una totalidad que articula identidades diferenciales y relacionales, es decir, identidades con un "carácter incompleto, abierto y políticamente negociable" ( $p$. 142). Ello no refiere a otra cosa más que al campo de la sobredeterminación, en el que la necesidad como tal solo "existe como limitación parcial del campo de la contingencia" (p. 151).

Antes de pasar a dar cuenta del lugar concreto que ocupa el concepto de "calidad educativa" en la totalidad discursiva que nos ocupa, quisiéramos dejar en claro que aunque nuestro análisis se sustente 
en documentos de políticas educativas, estas deben ser pensadas tan solo como algunos de los elementos que forman parte de ese discurso de la calidad de la educación. Junto a estos documentos, existen también otros objetos que se articulan y entran en relación a partir del discurso como, por ejemplo, ciertas instituciones (podemos pensar en el propio Estado, las universidades, los organismos creados para la evaluación de la calidad, etc.), los rituales del habla de quienes son considerados especialistas 0 , también, diferentes prácticas de órdenes diversos (como la evaluación, la investigación, la planificación, la enseñanza y el aprendizaje, etc.).

\section{La calidad educativa y el ser-educación}

$\mathrm{Si}$ el discurso no es una positividad dada y delimitada es porque la apertura de lo social es constitutiva y eso implica la imposibilidad de una fijación última del sentido. Sin embargo, el discurso se constituye como totalidad estructurada a partir de la práctica articulatoria. Esto significa que hay un intento de dominar el campo de la discursividad - exceso de sentido- o flujo de diferencias a partir de una fijación parcial del sentido. De este modo, Laclau y Moufee (2011) destacan la presencia de puntos discursivos privilegiados que permiten esa fijación parcial, a los que denominan puntos nodales.'

Ahora bien, para dar cuenta de los desplazamientos de sentido debemos adelantarnos en la exposición y señalar qué significa que haya puntos privilegiados en el discurso. De este modo, debemos indicar que cuando dijimos que el concepto de "calidad educativa" constituye el "centro" del discurso de la calidad de la educación, hacíamos referencia a que se constituye como un punto nodal, a partir del cual se va a organizar el sistema de relaciones diferenciales y a detenerse el deslizamiento de sentido. No obstante, el uso que hacemos de la palabra "centro" debe conllevar una formulación teórica a fin de

1 Esta noción teórica que proponen los autores es similar al concepto de "point de capiton" (basta de acolchado o punto de almohadillado) que desarrollara Jacques Lacan (2003). evitar una mala interpretación de la misma al emparentarla con un origen fijo. Tal como lo enuncia Derrida (1989), el centro no puede pensarse como un significado trascendental a partir del cual se funda una estructura, sino como una función. Lejos de pensar que se trata de un lugar fijo que responde a la determinación del ser como presencia, el autor sostiene que se trata de un no-lugar, imposible de buscar por fuera del propio discurso. En este sentido, la falta de centro o de fundamento nos introduce en el juego de la significación que no es otra cosa que el campo de la discursividad que mencionan Laclau y Moufee (2011). Pero para que se pueda dar la fijación parcial del sentido y detener su deslizamiento, producto de ese juego, es necesario, según Derrida, que esa falta se vea suplida por un signo que cumple la función de centro. Así, llegamos al punto nodal "calidad educativa", en tanto entendemos que se constituye cumpliendo tal función. Es a partir de este sintagma, entonces, que podemos pensar que se organiza, retroactiva y prospectivamente, todo lo que sucede en el discurso, sin perder de vista con ello que "todo discurso de la fijación pasa a ser metafórico: la literalidad es, en realidad, la primera de las metáforas" (p. 151).

Siguiendo a Lacan, podemos entender el concepto de "calidad educativa" como una metáfora ligada a la cuestión del ser (en nuestro caso, se trata del ser-educación), que condensa y unifica un conjunto de elementos dispersos, que a nuestros fines expositivos llamaremos "significaciones prescritas". Por su parte, Laclau sostiene que el ser de los objetos, lejos de ser una esencia fija, siempre se constituye socialmente al interior de un discurso, es decir, tiene un carácter histórico y contingente. De este modo, para poder dilucidar cómo se significa y se despliega históricamente el ser-educación, debemos tener en cuenta ese conjunto de "significaciones prescritas"

2 De aquí en más, nos referiremos con este sintagma al conjunto de atributos con que se concibe a la educación, en tanto se busca enfatizar el carácter performativo que tienen estos elementos, al constituirse como parte de la política educativa de un país. 
y los desplazamientos de sentido del discurso de la calidad de la educación. En este sentido, entramos en el plano de la articulación metonímica, que hace posible la transferencia de la significación y se constituye en la base sobre la que interviene la metáfora (Lacan, 2003).

\section{El proyecto neoliberal}

Si bien podemos apreciar la emergencia del discurso de la calidad de la educación, a partir de la articulación de un conjunto de instancias o elementos heterogéneos, hemos planteado la imposibilidad de que sea entendido como una configuración estable, dada y delimitada. En este sentido, hemos quedado advertidos sobre la presencia de otros discursos que lo deforman, así como del carácter abierto y políticamente negociable de los elementos articulados. Por consiguiente, si en un primer momento la "calidad educativa" que pregonaban los Estados de los países centrales se articula con las políticas económicas de corte keynesiano y la educación se significa como aquella que permite el progreso social y favorece el desarrollo económico, la "crisis económica mundial"3 de la década de los setenta y los cambios que le siguieron subvierten ese sentido de la educación. Sin embargo, en este punto es importante señalar que lo que sucede es una sobredeterminación de un conjunto de circunstancias nuevas que va a tener como resultado lo que Laclau y Mouffe (2011), siguiendo a Gramsci, denominan crisis orgánica, a partir de la que se intentará construir un nuevo bloque histórico o formación discursiva en la que se articule una pluralidad de aspectos económicos, sociales y culturales. Es a esta formación discursiva a la que nos estaremos refiriendo, de ahora en más, cuando aludamos al discurso neoliberal.

3 En los textos de historia, como en los documentos de política pública, es común hacer referencia a un origen económico de la crisis por la que se postula la recesión del "modelo económico de posguerra". De este modo, se localiza el punto de estallido en el año 1973, cuando se dispara el precio de petróleo, y se describe el momento en términos de una "estanflación": alto grado de inflación y estancamiento de la economía, con elevados índices de desempleo.
Excede los propósitos de este trabajo dar cuenta exhaustiva de todas las instancias nuevas que entran en el juego de reenvíos simbólicos; sin embargo, es necesario realizar algunos señalamientos al respecto ya que nos permiten un análisis más acabado de los nuevos sentidos que asume el concepto de "calidad educativa". De este modo, debemos recordar que en ese contexto de crisis y de cuestionamiento a las políticas keynesianas, las políticas públicas comienzan a articularse con teorías prekeynesianas y monetaristas, que defienden los sectores sociales más conservadores. En tal sentido, adquiere visibilidad la reacción teórica y política que desde los años cuarenta venían dirigiéndole estos sectores a la idea de un Estado intervencionista que limitara los mecanismos de mercado, en tanto esto era entendido como una amenaza a la libertad económica y política. Por consiguiente, la idea de un nuevo liberalismo va a ser considerada como la respuesta a la crisis, y como primeras medidas programáticas se buscará la reducción del alcance del Estado, la adopción de políticas de restricción de la emisión monetaria y del gasto fiscal, y la restauración de una tasa "natural" de desempleo. La implementación de estas políticas se volverá efectiva y hegemónica a finales de los años setenta (Anderson, 1999).

A consecuencia de ello, las instituciones económicas internacionales, paulatinamente, adquieren mayor incidencia en las decisiones políticas de los países en desarrollo. A nivel del Estado, esto no supone una falta de intervención en las medidas tomadas sino, por el contrario, un modo específico de actuación. Como veremos más adelante, esto tendrá como correlato para el área de educación, nuevas leyes en los diferentes países latinoamericanos. Asimismo, a lo largo del análisis podremos observar cómo, a nivel regional, diferentes documentos de política educativa generados en el marco de organismos internacionales como la Unesco reproducirán enunciados de aquellas instituciones de crédito.

Otra de las transformaciones que repercute en el discurso de la calidad educativa es el desmo- 
ronamiento de los modelos de producción fordista y taylorista y, en consecuencia, la declinación del liderazgo de Estados Unidos a nivel mundial. Como contrapartida, se afianza el nuevo modelo toyotista de producción, que venía desarrollando Japón como parte de su reconstrucción tras la derrota en la Segunda Guerra Mundial, asumiendo un rol protagónico en la industria avanzada basada en el valor añadido. A la par de este proceso, se configura una nueva concepción de la calidad, conocida como " $\mathrm{Ca}$ lidad total"4 que, a partir de la década de los ochenta, va a predominar en las empresas de todo el mundo. Debemos indicar, brevemente, que a partir de ese momento la "calidad" deja de concebirse como una herramienta de control para convertirse en una estrategia empresarial para el logro de una mayor competitividad. Entre sus características principales, supone la idea de que la calidad es el resultado del trabajo y de la participación de todos los inte-

4 Esta nueva concepción de la calidad, en la esfera económica, plantea una nueva estrategia de control que había sido empleada por el teórico Feigenbaum cuando trabajaba en la empresa General Electric. Fue este mismo autor quien la difunde a través de diversas publicaciones: La calidad como gestión (1945) y Total Quality Control (1951). A través de este nuevo enfoque se propone una coordinación interdepartamental y un nuevo tipo de administración de la organización. De este modo, la calidad pasa a ser el resultado del trabajo y la participación de todos los empleados, pero manteniendo una fuerte función gerencial que se encarga de facilitar el proceso sin perder de vista las necesidades del cliente (Márquez Gutiérrez, 2006). Asimismo, esta nueva estrategia empresarial se complementa con la idea esbozada por Deming y Juran referente a una "mejora continua" del proceso productivo. Debido a que todos estos teóricos habían llevado sus ideas a Japón, este es el primer país en implementar el nuevo enfoque, por ejemplo, en la empresa Toyota. De este modo, debemos señalar que diversos teóricos locales desarrollan ideas complementarias para adaptar este enfoque a su propia cultura. En este sentido, se destacan: Kaouro Ishikawa, quien adapta el concepto de Feigenbaum para el sistema administrativo de su país denominándolo Company Wide Ouality Control (CWOC), en alusión a un control total de toda la compañía (desde la vida personal de los empleados hasta la satisfacción de cliente); Shigeo Shingo y Taichi Ohno, creadores del "sistema toyotista"; y Masaaki Imai que, a través de la publicación de su libro Kaizen - La clave de la ventaja competitiva japonesa, sistematiza las ideas del modelo japonés y su diferencia con el occidental, señalando las diversas técnicas que se utilizan como, por ejemplo, el concepto de "Cero defectos", Just in time, Control Total de la Calidad, etc. grantes de la empresa, en tanto permite una "mejora continua" del producto o servicio. En este sentido, el factor humano ocupa un lugar central, por lo que se concibe al empleado como un ser autónomo con poder para controlar y ser responsable de la calidad, al tiempo que se busca su motivación y capacitación constante, junto al desarrollo de habilidades para el trabajo en equipoy la resolución de problemas, entre otras (Márquez Gutiérrez, 2006). El hecho de que la "calidad" deje de significarse como una herramienta de control y se convierta en una estrategia empresarial de repercusión mundial no es un dato menor,ya que, como veremos luego, su amplia divulgación no solo acompaña el auge que va adquiriendo la preocupación por la calidad educativa sino que, además, las ideas que la sustentan serán articuladas en y por el discurso de la calidad de la educación.

Si bien el discurso neoliberal no se reduce a estos elementos que hemos señalado, consideramos que los mismos nos ofrecen, por el momento, un marco de inteligibilidad suficiente para el abordaje de los desplazamientos de sentido del discurso de la calidad de la educación. No obstante, a medida que avancemos en el análisis iremos dando cuenta de otros elementos.

\section{La calidad como "falta"}

Como hemos sostenido desde un principio, el concepto de "calidad educativa" nos permite dilucidar un conjunto de propiedades o "significaciones prescritas" - como hemos preferido llamarlas-, que constituyen al ser-educación que sostiene la política educativa de un país. Ahora bien, a partir de los enunciados del discurso de la calidad de la educación se puede advertir que la calidad es construida siempre como una falta. Es decir, aunque las enunciaciones nos permitan acceder a ese conjunto de atributos que constituyen la identidad de la educación, estos elementos, la mayor parte de las veces, son exhibidos en tanto que carencias que impiden que el ser-educación sea como debiera ser. 
La falta de calidad, en tanto regularidad discursiva, es enunciada en términos de "pérdida" o "deficiencia" y siempre partiendo de un diagnóstico del presente educativo. En tal sentido, se construye un momento pasado en el que la calidad existía o era mejor. Sin embargo, esa construcción no supone un deseo de volver a lo que hubiera sido la calidad educativa alguna vez, es decir, sosteniendo las propiedades del ser-educación de una etapa anterior, sino que el pasado es el efecto de una práctica articulatoria actual en la que funciona como el referente de una realidad posible: el fin de la falta. De este modo, los enunciados evidencian la creencia en la consecución de las "significaciones prescritas" y, por consiguiente, en un estado pleno del ser-educación. Así, el diagnóstico sobre la falta de calidad opera como justificativo de la necesidad de la intervención del Estado en materia de educación - para su recuperación o mejoría-y, al mismo tiempo, legitima las acciones concretas que se lleven a cabo.

Ahora bien, el diagnóstico de una falta de calidad en el presente supone no solo un pasado en el que este problema no existiría, sino que también implica un momento de cambio a partir del cual se produce la "pérdida" o "deficiencia". En este sentido, la falta se significa como tal con base en dos circunstancias: la expansión del sistema educativo y el propio cambio histórico, ambas entendidas bajo el ideal de progreso, ya sea educativo, ya sea de las sociedades modernas. Es decir, en tanto idea o creencia que exige un acto de fe y que supone el movimiento de la historia en una dirección definida y deseable, ascendente y hacia adelante (Bury, 1971), en conformidad, principalmente, con el desarrollo de la economía, las ciencias y la tecnología. Estas dos circunstancias son articuladas en y por el discurso de la calidad educativa, y no solo permiten que la falta adquiera su significación, naturalizándola a partir de entender que los cambios que producen las deficiencias de la calidad son inevitables, puesto que son parte del progreso, sino que, además, se presentan como razón suficiente de una suerte de actualización de las "significaciones prescritas" de la educación.
Si bien estas dos situaciones que significan a la "falta de calidad" se presentan en el discurso como continuidades, son más evidentes al momento de producirse la crisis que mencionáramos más arriba. Y esto sucede porque la propia crisis es articulada con la idea de un "nuevo orden económico mundial" que, más allá de las tomas de posición que suponga al interior de un campo de relaciones de fuerza, es traducido por los actores sociales enunciadores como la necesidad de efectuar cambios a nivel educativo. De modo que, nuevamente, puede observarse de qué modo el diagnóstico de la "pérdida" o "deficiencia" de calidad opera justificando la intervención estatal. Pero ahora podemos precisar que tal acción estará sobredeterminada por la nueva formación discursiva neoliberal que se configura tras la crisis.

Para ilustrar lo que hemos planteado hasta aquí, consideremos los resultados de la Conferencia de Ministros de Educación de América Latina y Ministros encargados del Fomento de la Ciencia y de la Tecnología en relación con el Desarrollo en América Latina y el Caribe, de 1971, organizada por la Unesco en cooperación con la Comisión Económica para América Latina y el Caribe (Cepal) y la Organización de los Estados Americanos (OEA). Allí se plantea que si bien se produjo una expansión de los sistemas educativos, se registran ciertas "deficiencias de la educación", entre las que se enuncia "la calidad educativa". Esta es entendida en dos planos: "el de la infraestructura instrumental y los requisitos para una educación eficaz, y el de la orientación y conte-

$5 \quad$ El Informe Final de la Conferencia de Ministros, celebrada en Venezuela, manifiesta que si bien se ha impulsado la expansión de la educación primaria, promovida por el Proyecto Principal para la Extensión y el Mejoramiento de la Educación Primaria en América Latina, prevalecen "problemas mayores": déficit en la escolarización completa, analfabetismo, desequilibrios en las oportunidades de acceso, permanencia y progreso en los sistemas educativos, bajos niveles de rendimiento (medidos por los índices de deserción y repetición), deficiencias y desequilibrios estructurales de los sistemas educativos, la calidad de la educación y la necesidad de que el planeamiento supere su etapa de simple racionalización del desarrollo cuantitativo e integre otras dimensiones (Unesco, 1972, pp. 12-14). 
nido de la educación que se imparte". Respecto del primero, se hace alusión a la importancia que se le otorgó a la expansión cuantitativa, descuidando la infraestructura, los recursos didácticos de las escuelas y el personal docente. En cuanto al segundo plano, se mencionan tres cuestiones fundamentales: a) "la escasa vinculación del contenido de la educación con el trabajo y con su valor como elemento básico de la formación y el desarrollo del individuo y para su contribución a la construcción de la sociedad", proponiendo una "educación en el trabajo y para el trabajo"; b) "el escaso desarrollo que la ciencia como contenido y como instrumento de formación general tiene en los sistemas educativos", entendiendo que la misma es el motor del progreso y el medio para superar la dependencia; y c) se indica que debe promover una educación integral compuesta de una "formación intelectual, la valuación y las virtudes del trabajo, el desarrollo de capacidades creadoras, el sentido de la convivencia" (Unesco, 1972, p. 13).

Este ejemplo que hemos escogido no solo nos permite dar cuenta de lo que venimos sosteniendo respecto de la regularidad discursiva de la falta de calidad, sino que, además, a través de él podemos abordar la articulación metonímica de las "significaciones prescritas" que constituyen a la calidad educativa. De este modo, observamos que el sentido se desplaza entre "dos planos", que para nuestros fines expositivos sostenemos a fin de poder señalar esa "actualización" que advirtiéramos más arriba. Así, mientras que en el primero de estos planos, la "calidad educativa" se articula a cuestiones de índole material y que podríamos catalogar como exclusivamente escolares (por ejemplo, el salario docente y los recursos pedagógicos), el segundo evidencia el desplazamiento de sentido del discurso de la calidad educativa, abarcando nuevos elementos que vinculan la educación con el trabajo, la ciencia y el contexto mundial, a la par que señalan la importancia política que la calidad tiene para los Estados, en tanto se halla fuertemente ligada al futuro social y nacional de las poblaciones.
Asimismo, puede apreciarse en el ejemplo anterior que, en Latinoamérica, el diagnóstico de la falta de calidad se sustenta principalmente en la expansión del sistema educativo, y que el "contexto mundial" se presenta como el indicador de nuevas necesidades educativas. Distinto es lo que ocurre en los países desarrollados, ya que en estos el poder político se ampara en la nueva configuración mundial para diagnosticar el deterioro de la calidad y señalar los elementos del ser-educación. Dentro de este segundo grupo de naciones, nos debemos focalizar en el caso norteamericano, por ser uno de los primeros países en que los enunciados de los sectores políticos conservadores dan cuenta del desplazamiento de sentido de la educación a partir del discurso neoliberal. Veamos, entonces, cuáles son los elementos articulados.

En Estados Unidos, la crisis no solo conlleva un quiebre en el desarrollo económico sino, también, la pérdida de poder internacional frente a la creciente participación de Japón y los nuevos países industrializados de la zona del Pacífico. En este contexto, el conservadurismo pone en duda la idea de que "más educación" equivalga a "más progreso", tal como habían venido sosteniendo los sectores progresistas, y decide llevar a cabo diversos diagnósticos críticos $^{6}$ acerca de la calidad de los sistemas educativos. En un contexto de altos niveles de desempleo juvenil, estos diagnósticos coinciden en tres cuestiones: a) "en una crítica al sistema educativo por su inadecuación a las necesidades de los sectores de la producción y los servicios"; b) "en la baja rentabilidad individual (subempleo o desempleo de la mano de obra calificada) y social de la inversión educativa; y c) "bajos niveles de logros educativos en comparación con [...] otros países" (Diker y Feeney, 1998, p. 55). Un ejemplo por excelencia de estos diagnósticos es el

$6 \quad$ En Estados Unidos se realizan los siguientes estudios: A Nation at Risk (1983); American Competitive Challenge (1983); Academic Preparation for College (1983); Involvement in Learning (1984); To Reclaim a Legacy (1984), America 2000 and the politics and of reasure: democracy and cultural difference under siege (1992). 
informe Una nación en peligro: el imperativo de una reforma educacional (A Nation at Risk) 7 de 1983, que analiza la falta de calidad y elabora recomendaciones para su mejoría. La importancia de este documento es señalada por varios autores de la literatura especializada latinoamericana (Filmus, 1995; Casassus, 1999; Bianchetti, 2009), al punto de considerarlo el origen de la preocupación por la calidad educativa. El informe plantea que la pérdida de calidad pone en peligro la competitividad e integración social del país norteamericano (Casassus, 1999), en el advenimiento de una nueva "era informática", que implica una nueva distribución del conocimiento y una demanda de mano de obra altamente cualificada en nuevos campos (Gardner, 1983).

Según sostiene Bianchetti (2009), los grupos políticos conservadores de Estados Unidos toman las conclusiones del informe como un argumento para reformular y reorientar los contenidos de la educación y los modelos institucionales. Y esto, a su vez, implica una vuelta a los análisis que vinculan la economía con la educación, mediante la evaluación de la relación costo-beneficio (tanto a nivel individual como en relación con el crecimiento económico del país), expresado a través de la teoría del capital humano. El autor sostiene que esta teoría, en cuyo origen se halla la teoría del funcionalismo, "se sustenta sobre el principio de que la educación

$7 \quad$ El informe A Nation at Risk fue publicado por la Comisión Nacional de Excelencia en Educación de Estados Unidos, en 1983. Esta comisión había sido creada por el Secretario de Educación, T. H. Bell, bajo la presidencia de Reagan, con el fin de examinar la pérdida de la calidad de la educación y elaborar un informe con recomendaciones prácticas para la mejora de la educación y su reforma. Los objetivos que se planteó la Comisión fueron: 1) valorar la calidad del proceso de enseñanza y aprendizaje en las escuelas públicas y privadas, los colegios (colleges) y las universidades; 2) comparar las escuelas y colleges norteamericanos con otros de naciones industriales avanzadas; 3 ) estudiar la relación entre los requerimientos para la admisión en los colegios y el desempeño académico; 4) identificar los programas educativos que hayan generado logros académicos destacables; 5 ) evaluar la intensidad con que los cambios sociales y educacionales de los últimos 25 años han afectado el desempeño académico de los estudiantes; y, 6) definir los problemas por enfrentar y superar para alcanzar con éxito la excelencia en la educación (Gardner, 1983). constituye una inversión", que va a ser usada por los encargados de las políticas educativas. Estos van a considerar a la educación como aquella que prepara los recursos humanos demandados por los procesos productivos y, con base en el análisis de la relación costo-beneficio, y su consecuente escala de valores, van a "establecer prioridades en la toma de decisiones de Política Educacional [que] le otorgan el sentido originario al concepto 'calidad'” (p. 9). Estas nuevas ideas que plantea Estados Unidos para el campo de la educación, paulatinamente se van a ir expandiendo hacia el resto del mundo, junto al proyecto neoliberal, y gracias a la ayuda de los organismos multilaterales que financian, entre otros, a los países latinoamericanos.

\section{La calidad ausente y las reformas educativas}

Como señalamos en el apartado anterior, el continuo diagnóstico de la falta de calidad educativa opera justificando la necesidad de intervención estatal en el área, al tiempo que legitima las acciones concretas contraídas al respeto. En tal sentido, y siguiendo la lógica de causalidad que atraviesa los documentos analizados, nos hallamos con una segunda regularidad discursiva, la reforma. Así, cuando por primera vez la calidad educativa se formula como objetivo explícito de política pública para Latinoamérica, la misma es articulada al significante "reforma/s". Es lo que sucede cuando la Unesco aprueba, en 1980 y para los siguientes veinte años, el Proyecto Principal de Educación en América Latina y el Caribe (PPE), a pedido de los ministros de Educación de la región. Allí se establecen como objetivos específicos: alcanzar la escolarización básica, superar el analfabetismo y "mejorar la calidad y la eficiencia de los sistemas educativos a través de la realización de las reformas necesarias" (Unesco, 1981, p. 4). Esta formulación, asimismo, representa un indicador del lugar que adquiere la preocupación por la calidad, dejando de estar en un segundo plano respecto de la expansión del sistema educativo. 
Ahora bien, como hemos expresado, las acciones que llevan a cabo los diferentes Estados están sobredeterminadas por el discurso neoliberal. En este sentido, si la reforma educativa se dispone discursivamente como única respuesta posible a la falta de calidad, es porque lo hace al interior de un "proyecto de reforma ideológica", en el que se construye y difunde un nuevo sentido común, que "brinda coherencia, sentido y pretendida legitimidad a las propuestas de reforma impulsadas por el bloque dominante", como respuesta a la crisis capitalista (Gentili, 1999, p. 129).

Como habíamos anticipado, la mayoría de los documentos formulados a nivel regional, en el seno de organismos internacionales como la Unesco, no hacen más que reproducir los lineamientos neoliberales que formulan las instituciones de crédito. En este sentido, que la mejora de la calidad se articule a políticas que abogan por la eficiencia en el manejo de los recursos económicos tiene como condición de posibilidad las enunciaciones que el Banco Mundial formula como opciones de políticas para los países latinoamericanos. Tan es así, que incluso la sugerencia de la "asistencia social compensatoria a los educandos" como factor determinante de la calidad (Unesco, 1987, p. 43), se construye como una necesidad para evitar el posible fortalecimiento de desigualdades regionales, consecuencia de las reformas de regionalización y descentralización por las que, paradójicamente, se aclama para el logro de la mejora de la calidad y la eficiencia; reformas que, como veremos a continuación, habían sido ya impulsadas por el Banco Mundial. Antes, debemos señalar que estas reformas alusivas a cambios organizativos y administrativos implican un cambio de perspectiva que adopta diversas ideas de las nuevas ciencias empresariales posfordistas (autonomía o autogestión, competitividad, rendición de cuentas, calidad del servicio, satisfacción del cliente, etc.) y que provoca la incorporación de un nuevo término proveniente del campo de la economía, el de ges- tión. ${ }^{8}$ Asimismo, debemos destacar que las reformas son presentadas como una obligación a partir de "la actual situación de crisis financiera como [de] las demandas que genera el acelerado proceso de cambio científico-técnico y de democratización de la sociedad" (Unesco, 1988, p. 20).

A través de la publicación de El financiamiento de la educación en los países en desarrollo. Opciones de Políticas (Psacharopoulos, Tan y Jiménez, 1987), el Banco Mundial realiza una crítica de los sistemas de financiación de Latinoamérica al señalar que el gasto público en educación es "ineficaz". Luego, sugiere diferentes opciones de reforma que darían como resultado un aumento de recursos, una mejora en su utilización y un acceso más equitativo a la enseñanza. Entre estas se halla la opción de una descentralización, al entender que la actual fiscalización estricta que se realiza sobre las escuelas privadas y comunitarias les impide a estas contribuir al desarrollo de la educación. Por el contrario, el organismo sostiene que si se descentraliza y se les otorga mayor libertad, se movilizarán nuevos recursos privados, a la vez que mejorará la eficacia y eficiencia, al haber mayor competitividad entre la totalidad de las escuelas. La argumentación que sustenta estas ideas indica que con el aumento de la competencia se dará una mayor oferta de servicios educacionales, disminuyendo los costos y permitiendo que estudiantes y padres participen "en la elección de la calidad y el tipo de educación que desean y el modo de obtenerla" (p.4); esto, a su vez, haría que las escuelas aumenten su eficiencia, al exigírseles responsabilidades administrativas. Como puede percibirse, esta forma de pensar el sistema educativo está en consonancia con las

8 Si bien dicho concepto ya se usaba desde la década de los sesenta en Estados Unidos, en América Latina se consolida a fines de los años ochenta cuando dejan de considerarse la planificación y la administración como dos actividades separadas. Según Casassus, la separación respondía a una visión autoritaria o verticalista de la gestión (que solo era usada para hablar de administración o ejecución) propia de los sistemas educativos centralizados. Estas transformaciones paulatinas se acentúan y expanden en la región durante los años noventa. 
ideas del libre mercado, en tanto se sugiere que hay que otorgarle libertad a las escuelas para que compitan entre sí, entendiendo que esto necesariamente tendrá como resultado una mejora en la totalidad del sistema educativo. Así, ante la amenaza que representaría verse expuestas en la información que le suministraría el Gobierno central a la sociedad para que esta pueda elegir racionalmente lo que le conviene, las escuelas asumirían sus responsabilidades de ofrecer una educación de calidad.

\section{La calidad educativa como problema global}

Si bien hasta aquí hemos dado cuenta de los desplazamientos de sentido de la calidad educativa a partir de la sobredeterminación del proyecto neoliberal, señalando dos regularidades discursivas (el diagnóstico de la falta de calidad y la reforma para su mejoría), el análisis se ha focalizado, principalmente, en el momento en que se produce la crisis y se construye discursivamente la idea de un "nuevo orden económico internacional". Por el contrario, si ahora nos centramos en el momento en que el discurso neoliberal se vuelve hegemónico, nos hallamos con un discurso de la calidad de la educación en el que las "significaciones prescritas" abordadas adquieren nuevos sentidos, al articularse con otras. Ahora bien, para dar cuenta de esta transferencia de la significación, es preciso que primero indaguemos en aquellas instancias históricas que entran en el juego de reenvíos simbólicos que hacen que el neoliberalismo se construya como el único discurso posible capaz de dar respuesta a la crisis.

En este sentido, Perry Anderson (1999) sostiene que a inicios de los años noventa el capitalismo avanzado entra en una nueva crisis que, sin embargo, no logra suscitar una reacción violenta en contra del neoliberalismo. Por el contrario, señala que este consigue un segundo impulso cuando la caída del comunismo y el fin de la Guerra Fría conllevan su expansión hacia Oriente, al tiempo que la crisis hiperinflacionaria en América Latina encuentra como única respuesta posible las políticas neoliberales. De este modo, el autor sostiene que, desde el punto de vista político e ideológico, el neoliberalismo se vuelve hegemónico, "diseminando la simple idea de que no hay alternativas para sus principios, y que todos, partidarios u opositores, tienen que adaptarse a sus normas" (p. 27).

Con este nuevo contexto político internacional, aquello que había sido concebido como el advenimiento de un nuevo orden internacional, comienza a ser enunciado mediante los significantes "globalización”, “mundialización” o "interdependencia”. En líneas generales, estos términos condensan aspectos económicos, políticos, sociales y culturales con un presunto carácter global que, como veremos, subvierten el sentido del discurso de la calidad de la educación. Así, la "globalización" operará justificando y naturalizando la hegemonía neoliberal, especialmente en relación con aquellos acontecimientos discursivos que podemos ligar a lo que hemos denominado "esfera económica". Y, al mismo tiempo, este significante se articulará -y se complementará-, con el sintagma "sociedad del conocimiento", a partir del que se sintetizan aspectos culturales, ligados principalmente a "un nuevo paradigma tecnológico que altera y modifica los procesos organizacionales en la relación capital-trabajo" (García Delgado, 1994, p. 57), lo cual hace que la acumulación del capital dependa cada vez más de la intensidad del conocimiento y, por consiguiente, provoque la "desmaterialización" del trabajo. En este sentido, recordemos que en los años ochenta, como parte de su diagnóstico de la pérdida de calidad, las autoridades norteamericanas ya anunciaban una "era informática" y la importancia de los aprendizajes en ciencia y tecnología. Como habíamos anticipado en ese momento, las ideas que aquel país planteaba para la mejora de la calidad, vinculadas al funcionalismo y a la teoría del capital humano, fueron gradualmente adoptadas por los Estados latinoamericanos, ocupando un rol central en ello los organismos multilaterales de crédito. De este modo, aunque la falta de calidad 
siempre sea articulada en América Latina a la expansión del sistema educativo y al contexto mundial, signado por el desarrollo de la economía, de la ciencia y de la tecnología, esta última circunstancia adquiere mayor relevancia respecto de la primera, una vez que el neoliberalismo se torna hegemónico.

Como veremos a continuación, todo esto lleva a que el concepto de "calidad educativa" se articule con mayor cantidad de "significaciones prescritas", al tiempo que el discurso de la calidad de la educación domina el exceso de sentido que supone la discursividad de lo educativo, convirtiéndose esa fijación parcial del sentido en el modo legítimo -y normativo- del ser-educación en la actualidad.

\section{Calidad y desarrollo en educación ${ }^{9}$}

Anteriormente, señalamos que la reforma educativa se construye discursivamente como la herramienta política que tienen los Estados para mejorar la calidad. En este sentido, hemos abordado cómo al interior de esa reforma se buscan establecer cambios de índole organizativa y administrativa que suponen una mejora de la eficiencia del sistema educativo. Sin embargo, en esa ocasión nos hemos focalizado tan solo en algunos de los significantes que articula el discurso pero, tal como señalan las propias enunciaciones, "las transformaciones en la gestión [...] son condiciones necesarias pero no suficientes de la nueva estrategia educativa" (Unesco, 1991, p. 45). De este modo, si ahora nos centramos en los sintagmas "calidad educativa" o "mejora de la calidad", podemos dilucidar las otras partes de ese conjunto de "significaciones prescritas" con el que se define el ser-educación.

9 En el marco de la IV Reunión del Comité Regional Intergubernamental del Proyecto Principal de Educación, convocada por la Unesco, los ministros de Educación reconocen que "las estrategias tradicionales en que se han sustentado los sistemas educativos de la región han agotado sus posibilidades de armonizar cantidad con calidad. Sostenemos, en consecuencia, que estamos en un momento de enorme trascendencia histórica, definido por la necesidad de iniciar una nueva etapa de desarrollo educativo, que responda a los desafíos de la transformación productiva, de la equidad social y de la democratización política" (Unesco, 1991, p. 44).
En tal sentido, debemos señalar que la mejora de la calidad supone también una reforma de las prácticas pedagógicas, articulándola a "procesos de profesionalización docente" y a una "transformación curricular", basada en "la satisfacción de las necesidades educativas básicas del individuo y de la sociedad" (Unesco, 1991, p. 45). La idea de una "educación básica [que] debe centrarse en las adquisiciones y los resultados efectivos del aprendizaje, en vez de prestar exclusivamente atención al hecho de matricularse" (Unesco, 1990, p. 6), conforma el núcleo del proyecto educativo mundial acordado en la Conferencia Mundial sobre Educación para Todos (en adelante, proyecto EPT). ${ }^{10}$ Si tenemos en cuenta que este encuentro supone el consenso de la mayoría de los Estados y que, por consiguiente, el proyecto posee legitimidad mundial, puede entenderse por qué, a pesar de que allí no hay una definición del concepto de "calidad educativa", dos de los elementos que se enuncian como claves para el área se vuelven centrales en la práctica articulatoria: la determinación de niveles aceptables de adquisición de conocimientos en los planes de educación y la aplicación de sistemas de evaluación de los resultados.

En tal sentido, por ejemplo, con la sanción de la Ley Federal de Educación argentina (LFE 24.195, 1993), se incorpora el concepto de "calidad de la educación" como equivalente al de "calidad de la formación", y se señala que esta estará garantizada mediante la evaluación permanente del sistema educativo (art. 48). Asimismo, se añade que esa evaluación "verificará la adecuación de los contenidos curriculares [...] a las necesidades sociales y a los requerimientos educativos de la comunidad, así como el nivel de aprendizaje de los alumnos/as y la calidad de la formación docente" (art. 49).

10 La conferencia fue convocada por los jefes ejecutivos del Fondo de las Naciones Unidas para la Infancia (Unicef), el Programa de las Naciones Unidas para el Desarrollo (PNUD), la Organización de las Naciones Unidas para la Educación, la Ciencia y la Cultura (Unesco) y el Banco Mundial. Como resultado de la reunión se produjeron dos documentos: la "Declaración mundial de educación para todos" y el "Marco de acción para satisfacer las necesidades básicas de aprendizaje". 
Ahora bien, si nos detenemos en cuáles serían esas "necesidades y requerimientos sociales", estos se despliegan a partir del sintagma "sociedad del conocimiento", el que, a su vez, es equivalente al de "sociedad moderna". En este sentido, los enunciados localizan a América Latina por fuera de aquella sociedad moderna y a la educación como una llave para su acceso:

Para superar la crisis económica e incorporarse al mundo moderno como protagonistas activos, los paises de la región necesitan robustecer su integración regional y sus vínculos bilaterales, invertir prioritariamente en la formación de sus recursos humanos y fortalecer su cohesión social. Sin educación de calidad no habrá crecimiento, equidad ni democracia (Unesco, 1991, p. 44).

De este modo, se construye discursivamente la idea de que es indispensable implementar un nuevo desarrollo educativo (para el que son necesarias las reformas), que contribuya al crecimiento o desarrollo económico de las naciones y, por consiguiente, a su inserción en el "mundo moderno" o en la "economía mundial". Esto supone la articulación entre educación y economía y, por ello, la intervención estatal para la mejora de la calidad ya no es solo pensada como política social sino que se considera tema de política económica. Se plantea así "establecer políticas que vinculen el sistema educativo con la industria y apoyar los esfuerzos de las empresas en materia de capacitación", tomando como ejemplo la experiencia de Japón (Unesco/Cepal, 1996, p. 49). En este sentido, ciertas ideas subyacentes al modelo toyotista de producción y al concepto de "Calidad total" del mundo empresarial son asumidas como válidas y se yuxtaponen con las del concepto de "calidad educativa"."

11 Al respecto debemos recordar que la idea de la descentralización del sistema educativo se articula con una nueva gestión educativa. Esta última supone, por un lado, una mayor autonomía de las escuelas y, por el otro, la responsabilidad por sus resultados internos. Tal como plantea el modelo toyotista y el enfoque de la "Calidad total", la calidad pasa a ser el resultado del trabajo y la participación de
En línea con lo anterior, en tanto las "deficiencias en la calidad" que se buscan revertir son, por lo general, presentadas a partir de una inadecuación entre el sistema de formación de recursos humanos y las necesidades del desarrollo, se plantea la inversión en capital humano como prioridad para una mayor productividad y competitividad. Lo que se enuncia es la obsolescencia de un modelo de desarrollo basado en la exportación de productos básicos y la industrialización sustitutiva de importaciones, y la necesidad de una "transformación productiva con equidad" a partir de considerar como factor principal el "progreso técnico". De este modo, "se requiere en el próximo decenio, un cambio hacia un sistema que privilegie la calidad de la enseñanza, la difusión del conocimiento y la relación fluida de la producción del conocimiento con la economía" (Unesco/Cepal, 1996, p. 36) para "contar con recursos humanos en buenas condiciones y con capacidad de agregar progresivamente valor intelectual y progreso técnico a su base de recursos naturales" (p.18).

A partir de considerar a la educación una "inversión social" y articulada con el "desarrollo" y el "progreso técnico", entonces se construyen como significaciones prescritas centrales en la definición de la calidad educativa a la "eficiencia", la "equidad" (como equivalente a la igualdad de oportunidades y articulada a las políticas compensatorias), la "formación para el trabajo", la "responsabilidad social por la educación", la "extensión de la escolarización", la "financiación privada de la educación", la "evaluación del rendimiento", la "descentralización" y la "educación continua".

Como señalamos, la determinación de niveles aceptables de adquisición de conocimientos en los planes de educación y la aplicación de sistemas de evaluación de los resultados se constituyen como

todos (trabajo en equipo). De este modo, la eficiencia y la participación de toda la comunidad escolar (directores, docentes, alumnos, padres), junto a la evaluación permanente, se traducen en una "mejora continua", núcleo del concepto de "Calidad total". 
los elementos centrales en el discurso de la calidad de la educación. Asimismo, hemos dado cuenta del lugar que ocupan ciertos elementos que podrían clasificarse como pertenecientes a la esfera económica. En este sentido, debemos resaltar que estos tres factores forman parte de lo que las enunciaciones del Banco Mundial (1996) llaman "orientación a los resultados", a través de la cual "las prioridades de la educación se determinan mediante el análisis económico, ${ }^{12}$ el establecimiento de normas y la medición del cumplimiento de las normas" (p. 104).

Así, por ejemplo, en las enunciaciones de la Cepal y la Unesco se señala que:

Los resultados que constituyen exigencias para el desempeño en el mercado de trabajo y los que son requisitos para el desempeño ciudadano, coinciden en muchos aspectos, constituyendo un cuerpo común de aprendizajes requeridos, basado en el dominio de los códigos culturales básicos de la modernidad y en el desarrollo de las capacidades humanas para resolver problemas, tomar decisiones y seguir aprendiendo (Unesco/Cepal, 1996, p. 43).

En esta misma línea, el Banco Mundial (1996) sostiene que "la enseñanza básica constituye la máxima prioridad en todos los países porque proporciona las aptitudes y conocimientos básicos necesarios para el orden cívico y para la plena participación en la sociedad, así como para todas las formas de trabajo" (p. 105). De este modo, a partir de los enunciados podemos dilucidar que la calidad educativa se articula con la "economía", a través de la formación de recursos humanos; con la "democracia", mediante la formación ética y ciudadana; $y$,

12 "El análisis económico aplicado a la educación se centra en la comparación entre los beneficios y los costos, para las personas y para la sociedad en conjunto. Se comparan los costos de las distintas intervenciones para lograr un objetivo educacional determinado y se mide la relación entre los beneficios y los costos, generalmente calculando la tasa de rentabilidad y considerando como beneficio la mayor productividad de la mano de obra, medida por las diferencias de salarios" (Banco Mundial, 1996, p. 105). asimismo, con los "códigos de la modernidad", entendiéndose por estos últimos "los lenguajes y competencias básicas para participar en la vida pública y productiva" (Unesco, 1996, p. 29).

\section{Conclusión}

A partir del análisis de la configuración del discurso de la calidad de la educación hemos podido observar cómo se fue estructurando un sistema de elementos diversos a través del cual fue posible dilucidar el modo particular en que la educación se significa al articularse con aquellos elementos, fijando provisoriamente su sentido y vinculándose con determinado ordenamiento social.

De este modo, hemos centrado nuestra atención en la sobredeterminación que supuso para las políticas educativas de las últimas décadas del siglo XX, la instauración del discurso neoliberal, articulando una pluralidad de aspectos económicos, sociales y culturales nuevos. Así, la exploración de los sentidos del sintagma "calidad educativa" nos permitió dar cuenta de dos regularidades discursivas: el diagnóstico de la "falta de calidad" y la consecuente necesidad de una "reforma educativa". En tal sentido, hemos señalado cómo ambos elementos operaron justificando y legitimando un modo específico de actuación por parte de los Estados en materia de educación, solidario con el orden neoliberal que se estaba instaurando.

De igual modo, al centrarnos en el momento en que el discurso neoliberal se volvió hegemónico y a partir del que finalmente se concretaron las reformas educativas en Latinoamérica, hemos advertido cómo el discurso de la calidad educativa significó a la educación a partir de su articulación con el término "globalización", que opera naturalizando aquella hegemonía a nivel mundial y, al mismo tiempo y complementariamente, junto al sintagma "sociedad del conocimiento", a partir del que se sintetizan aspectos culturales, ligados a la "desmaterialización" del trabajo humano y al progreso tecnológico. 
Como parte de este proceso, hemos evidenciado la relación que se establecía en América Latina entre la mejora de la calidad educativa y las ideas del funcionalismo, en particular la teoría del capital humano, reproduciendo el modelo norteamericano de reforma educativa, tal como Estados Unidos y los organismos multilaterales de crédito se habían propuesto. En tal sentido, las deficiencias de calidad en los países latinoamericanos dejaron de diagnosticarse principalmente como resultado de la expansión del sistema educativo y pasaron a estar vinculadas con un retraso regional respecto al desarrollo global de la economía, la ciencia y la tecnología.
Consecuentemente, la necesidad del desarrollo educativo se articuló a dos elementos claves: el aprendizaje de los conocimientos y de las competencias básicas, y la evaluación permanente de los mismos, respondiendo a presuntas exigencias globales para el adecuado desempeño laboral y ciudadano en las sociedades modernas.

De este modo, el discurso de la calidad de la educación pasó a dominar todo el campo educativo, fijando un sentido que hasta hoy se sostiene como el modo legítimo - y normativo- de la educación, que tiene entre sus consecuencias político-sociales más evidentes el sostenimiento del orden neoliberal.

\section{Referencias}

Anderson, P. (1999). Neoliberalismo: un balance provisorio. En Sader, E. y Gentilli, P. (comps.). La trama del neoliberalismo. Mercado, crisis y exclusión social (pp. 143-147). Buenos Aires: Clacso-Eudeba.

Banco Mundial (1996). Prioridades y estrategias para la educación:examen del Banco Mundial. Washington, DC: Banco Mundial. Recuperado el 27 de agosto de 2013 de http://www-wds.worldbank.org/external/default/WDSContentServer/WDSP/IB/2005/06/13/000160016_20050613172136/Rendered/PDF/14948010spanish.pdf

Bianchetti, R. (2009). Educación de calidad: uno de los dilemas fundamentales para las políticas educativas que se propongan demostrar, que "otro mundo es posible". Boletín del Foro Latinoamericano de Políticas Educativas, 6 (26), 1-28. Recuperado el 27 de agosto de 2013 de http://biblioteca.clacso.edu.ar/gsdl/collect/ ar/ar-033/index/assoc/D589.dir/2.pdf

Bury, J. (1971). Introducción. En La idea de progreso (pp. 13-42). Madrid: Alianza.

Casassus, J. (1999). Boletín Proyecto Principal de Educación 50 (pp. 47-65). Recuperado el 27 de agosto de 2013 de http://unesdoc.unesco.org/images/o011/o01191/119117s.pdf

Derrida, J. (1989). La estructura, el signo y el juego en el discurso de las ciencias humanas. En La escritura y la diferencia (pp. 383-401). Barcelona: Anthropos.

Diker, G. y Feeney, S. (1998). La evaluación de la calidad. Un análisis del discurso oficial. Revista del Instituto de Investigaciones en Ciencias de la Educación, 7 (12), pp. 55-63.

Filmus, D. (comp.) (1995). Los condicionantes de la calidad educativa. Buenos Aires: Novedades Educativas.

García Delgado, D. (1994). Del Estado de bienestar al Estado postsocial. En Estado y Sociedad (pp. 27-63). Buenos Aires: Tesis Norma.

Gardner, D. et al. (1983). A Nation At Risk: The Imperative For Educational Reform. Washington, DC: National Commission on Excellence in Education. 
Gentilli, P. (1999). Mentiras que parecen verdades: argumentos neoliberales sobre la crisis educativa. En Análisis político y propuestas pedagógicas (pp.129-138). Buenos Aires: Aique.

Lacan, J. (2003). La instancia de la letra. En Escritos I (pp. 473-509). México: Siglo XXI Editores.

Laclau, E. y Mouffe, C. (2011). Hegemonía y estrategia socialista. Hacia una radicalización de la democracia. Buenos Aires: Fondo de Cultura Económica.

Ley Federal de Educación 24195. Diario Oficial de la República Argentina, Buenos Aires, 4 de abril de 1993.

Márquez Gutiérrez, A. (2006). Calidad en las organizaciones: fundamentos, análisis y reflexiones. México: Universidad de Occidente. Recuperado el o3 de septiembre de 2013 de http://www.slideshare.net/tecnomexico/ calidad-en-las-organizaciones

Psacharopoulos, G., Tan, J. P. y Jiménez, E. (1987). El financiamiento de la educación en los países en desarrollo. Opciones de Políticas (Informe 8226). Washington, DC: Banco Mundial. Recuperado el o3 de septiembre de 2013 de http://www-wds.worldbank.org/external/default/WDSContentServer/WDSP/IB/2010/06/10/00 0333037_20100610023527/Rendered/PDF/82260PUBoSPANISHoBox70021B01PUBLIC1.pdf

Unesco (1972). Conferencia de ministros de Educación y de ministros encargados del fomento de la ciencia y de la tecnología en relación con el desarrollo en América Latina y el Caribe convocada en cooperación con la Cepal y la OEA. Paris: Unesco. Recuperado el 24 de septiembre de 2013 de http://unesdoc.unesco.org/ images/0000/000015/001524sb.pdf

Unesco (1981). Recomendación de Quito. Reunión regional intergubernamental sobre los objetivos, las estrategias y las modalidades de acción de un proyecto principal en la esfera de la educación en la región de América Latina y el Caribe. Quito: Unesco. Recuperado el 24 de septiembre de 2013 de http://unesdoc.unesco.org/ images/0015/001599/159981s.pdf

Unesco (1987). Informe final. Segunda Reunión del Comité Regional Intergubernamental del Proyecto Principal en la Esfera de la Educación de América Latina y el Caribe. Bogotá: Unesco. Recuperado de http://unesdoc. unesco.org/images/0007/000763/076303sb.pdf

Unesco (1988). Proyecto principal de educación en América Latina y el Caribe 19. Santiago de Chile: Unesco. Recuperado el 15 de junio de 2013 de http://unesdoc.unesco.org/images/00o8/000839/083983s.pdf

Unesco (1990). Declaración mundial sobre educación para todos y Marco de acción para satisfacer las necesidades básicas de aprendizaje. Jomtien: Unesco. Recuperado el 23 de agosto de 2013 el 15 de junio de 2013 de http://unesdoc.unesco.org/images/0008/000862/08628gsb.pdf

Unesco (1991). Proyecto principal de educación en América Latina y el Caribe 24. Santiago de Chile: Unesco. Recuperado el 23 de agosto de 2013 de http://unesdoc.unesco.org/images/ooog/000905/090515s.pdf

Unesco (1996). Informe final. Séptima Reunión de Ministros de Educación de América Latina y el Caribe. Kingston: Unesco. Recuperado el 23 de agosto de $2013 \mathrm{de} \mathrm{http://unesdoc.unesco.org/images/o011/001121/112197s.pdf}$

Unesco/Cepal (1996). Educación y conocimiento: eje de la transformación productiva con equidad. Lima: Unesco-Tarea. Recuperado el 12 de septiembre de $2013 \mathrm{de} \mathrm{http://unesdoc.unesco.org/images/0015/001502/1502535o.pdf}$ 
